\title{
Impacts des Hydrocarbures Aromatiques Polycycliques sur les poissons: Cas des Tilapias du lac Nokoué au Sud du Bénin (Afrique de l'Ouest)
}

Flavien Edia Dovonou, (PhD, Maître-Assistant)

Laboratoire d'Hydrologie Appliquée (LHA) à l'Institut National de l'Eau de l'Université d'Abomey-Calavi, Cotonou, Bénin

Moudachirou Ibikounle, (PhD, Maître de Conférences)

Laboratoire de Zoologie de la Faculté des Sciences et Techniques,

Université d'Abomey-Calavi, Cotonou, Bénin

Coovi Guénolé Akouedegni, (PhD, Maître-Assistant)

Laboratoire d'Ethnopharmacologie et de Santé Animale de la Faculté des

Sciences Agronomiques de l'Université d'Abomey-Calavi, Cotonou, Bénin

Valdina Aissi, (Phd Student)

Mahutondji Prudence Dossou, (Phd Student, Msc)

Daouda Mama, (Phd, Professeur Titulaire)

Laboratoire d'Hydrologie Appliquée (LHA) à l'Institut National de l'Eau de

l’Université d'Abomey-Calavi, Cotonou, Bénin

Doi:10.19044/esj.2019.v15n36p458 URL:http://dx.doi.org/10.19044/esj.2019.v15n36p458

Résumé

Les résultats issus des analyses physico-chimiques et toxicologiques des eaux et des poissons du lac Nokoué nous ont permis de constater que les Hydrocarbures Aromatiques Polycycliques retrouvés dans le lac influencent sensiblement la qualité des poissons et que les valeurs limites sont dépassées pour certains paramètres. Les valeurs de conductivité moyenne $(2752 \mu \mathrm{S} / \mathrm{cm})$ et de concentration moyenne des nitrites $(7,78 \mathrm{mg} / \mathrm{L})$ ont montré que les échantillons d'eau prélevés sur le lac Nokoué sont pollués. Le dosage des polluants a permis de constater que les individus de Tilapia guineensis, Hemichromis fasciatus et de Sarotherodon melanotheron prélevés dans les eaux du lac sont respectivement contaminés à $10 \%, 18 \%$ et $10 \%$ au niveau de la rivière Sô à Dogodo, à 26\%, 25\% et 23\% à Kétonou, 32\%, 29\% et $40 \%$ à $6 \mathrm{~km}$ de Domèguédji et 32\%, 28\%, 27\% à l'embarcadère d'Abomey-Calavi.

Mots clés: Lac Nokoué, Pollution, Produits Pétroliers, HAP Pétrogéniques, Tilapias 


\title{
Impacts of Polycyclic Aromatic Hydrocarbons on Fish: Case of Tilapias of Lake Nokoué in South Bénin (West Africa)
}

Flavien Edia Dovonou, (PhD, Maître-Assistant)

Laboratoire d'Hydrologie Appliquée (LHA) à l'Institut National de l'Eau de l'Université d'Abomey-Calavi, Cotonou, Bénin

Moudachirou Ibikounle, (PhD, Maître de Conférences)

Laboratoire de Zoologie de la Faculté des Sciences et Techniques,

Université d'Abomey-Calavi, Cotonou, Bénin

Coovi Guénolé Akouedegni, (PhD, Maître-Assistant)

Laboratoire d'Ethnopharmacologie et de Santé Animale de la Faculté des

Sciences Agronomiques de l'Université d'Abomey-Calavi, Cotonou, Bénin

Valdina Aissi, (Phd Student)

Mahutondji Prudence Dossou, (Phd Student, Msc)

Daouda Mama, (Phd, Professeur Titulaire)

Laboratoire d'Hydrologie Appliquée (LHA) à l'Institut National de l'Eau de

l’Université d'Abomey-Calavi, Cotonou, Bénin

\begin{abstract}
The results from physico-chemical and toxicological analyzes of the waters and fish of lake Nokoué have shown that the Polycyclic Aromatic Hydrocarbons found in the lake have a significant influence on the quality of the fish and that the limit values are exceeded for certain parameters. The mean conductivity and mean concentration values of the nitrites, estimated at 2752 $\mu \mathrm{S} / \mathrm{cm}$ at $7.78 \mathrm{mg} / \mathrm{L}$ respectively, show that the water samples taken from lake Nokoué are polluted. The bioaccumulation factor revealed that individuals of Tilapia guineensis, Hemichromis fasciatus and Sarotherodon melanotheron taken from lake waters are respectively $10 \%, 18 \%$ and $10 \%$ contaminated at the Sô River level in Dogodo, at 26\%, 25\% and $23 \%$ at Kétonou, 32\%, 29\% and 40\% at $6 \mathrm{~km}$ from Domèguédji and 32\%, 28\%, 27\% at the Abomey-Calavi jetty.
\end{abstract}

Keywords: Lake Nokoué Pollution, Petroleum Products, Petrogenic Pahs, Tilapias 


\section{Introduction}

La dégradation des écosystèmes aquatiques devient de plus en plus avancée avec le temps selon Anfray et al (2016). Nos plans et cours d'eau deviennent de plus en plus pollués faute d'une mauvaise gestion Baechler (2015). Cet état de chose est en fait lié la plupart du temps aux activités anthropiques menées aux abords de ces cours d'eau (Anderson, 2007). La République du Bénin n'est pas épargnée par ces problèmes, notamment la pollution liée aux transports des produits pétroliers sur les plans d'eau en général et sur le lac Nokoué en particulier (Tossou, 2001). L'une des activités caractéristiques menées sur le lac Nokoué est le transport des produits pétroliers, ainsi que l'utilisation des barques motorisées (Hoteyi, 2014). Ces activités engendrent plusieurs implications qui influencent négativement les écosystèmes naturels, ce qui pose un problème de santé publique (Dovonou, 2008). En effet, ces produits pétroliers contiennent diverses sortes de polluants tels que les HAP (Hydrocarbures Aromatiques Polycycliques) pétrogéniques qui ont un caractère lipophile leur permettant d'être transférés au sein des réserves lipidiques et dans les membranes cellulaires des organismes (Roussel, 2002). Ils peuvent également avoir des conséquences sur les comportements, l'alimentation et la reproduction des espèces aquatiques (Baussan, 2000). Leur présence dans la chair des espèces aquatiques consommées par les populations constitue un danger pour la santé de ces derniers car ils sont de nature cancérogène et peuvent entraîner des tumeurs de poumons, de foie, de la vessie et de la peau chez l'Homme (CIRC, 2012). Il urge de ce fait de mettre en œuvre une série de techniques et de méthodes visant à contrôler, surveiller et évaluer la pollution liée à ces hydrocarbures afin de prévenir les conséquences écologiques désastreuses pour la santé des espèces aquatiques ainsi que pour la santé de la population.

Le lac Nokoué, considéré comme le plus grand lac du Bénin couvre une superficie de $150 \mathrm{~km}^{2}$ à l'étiage (Dovonou, 2008). Situé au Sud-Est du pays, il est compris entre les parallèles $6^{\circ} 20$ et $6^{\circ} 30$ Nord et les méridiens $2^{\circ} 20$ et $2^{\circ} 35$ Est. S'étendant sur les départements de l'Ouémé, de l'Atlantique et du Littoral, il est limité à l'Ouest par le plateau d'Abomey-Calavi, à 1'Est par la lagune de Porto- Novo, au Nord par la plaine d'inondation du fleuve Ouémé et de la rivière Sô puis au Sud par la ville de Cotonou (Hounsinou, 2015). Le chenal de Cotonou et le canal de Totchè relient le lac respectivement à l'océan Atlantique et à la lagune de Porto-Novo (Fiogbé, 2009). Le lac Nokoué a une longueur moyenne de $20 \mathrm{~km}$ dans sa direction Est-Ouest et une largeur de 11 $\mathrm{km}$ dans sa direction Nord-Sud (Dovonou, 2012). (Figure 1). 


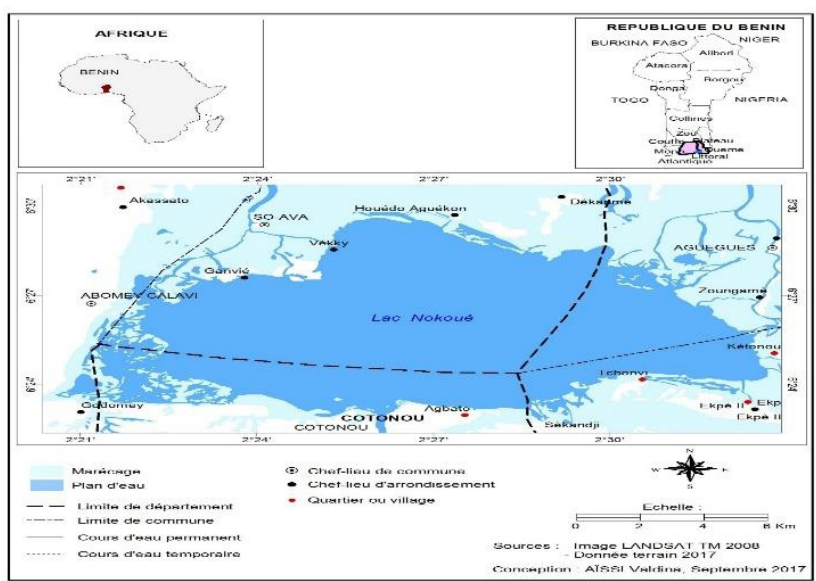

Figure 1 : Localisation du lac Nokoué au Bénin.

\section{Méthodologie}

La méthodologie adoptée pour cette étude est structurée en trois étapes. Le choix préalable des sites d'échantillonnages ; Les travaux de terrain pour la collecte des échantillons d'eau et de poissons au niveau du lac Nokoué et la rivière Sô et enfin l'analyse et le traitement des données.

\subsection{Le choix des sites d'échantillonnages}

Les sites d'échantillonnage sont le lac Nokoué et la rivière Sô (Figure 1). Le lac Nokoué étant le cadre de notre étude supposé contaminer et la rivière Sô est le site témoin. Sur le lac Nokoué, il s'effectue des transports de produits pétroliers, mais par contre, il ne se passe rien comme activités pétrolières sur la rivière Sô. Ces deux sites permettent de faire une comparaison sur la qualité des poissons du genre Tilapia qui sont très consommés par les populations $d u$ Sud-Bénin. Les prélèvements se sont effectués en Novembre 2017. Sur le lac Nokoué, trois sites A, B et C ont été sélectionnés. Le site A est le village Kétonou, le site $\mathrm{B}$ se situe à $6 \mathrm{~km}$ du village Domèguédji et le site $\mathrm{C}$ est l'embarcadère d'Abomey-Calavi. Trois points ont été sélectionnés sur chaque site pour des prélèvements d'eau et de capture de poissons. Sur la rivière Sô, trois points ont été sélectionnés à Dogodo pour des prélèvements d'eau et de capture de poissons : soit neuf (09) échantillons d'eau prélevés au total sur le lac Nokoué et trois (03) échantillons d'eau sur la rivière Sô.

Les sites choisis pour l'échantillonnage des poissons sont les mêmes sur lesquels ont été fait les prélèvements d'eau. (Figure 2). Trois espèces de poissons ont été récoltées au niveau de chaque point (03 points à Dogodo sur la rivière Sô, 03 points à l'embarcadère, 03 points à $6 \mathrm{~km}$ de Domèguédji et 03 points à Kétonou); soient 09 poissons à Dogodo sur la rivière Sô, 09 poissons à l'embarcadère d'Abomey-Calavi, 09 poissons sur le site B à $6 \mathrm{~km}$ de Domèguédji et 09 poissons à Kétonou. Au total, 36 poissons ont été 
prélevés sur les quatre stations citées plus haut dont 12 Tilapia guineensis, 12 Hemichromis fasciatus et 12 Sarotherodon melanotheron.

\subsection{Les travaux de terrain}

Les prélèvements ont été effectués sur le lac Nokoué (sur le site A à Kétonou, sur le site $\mathrm{B}$ à $6 \mathrm{~km}$ de Domèguédji et sur le site $\mathrm{C}$ à l'embarcadère d'Abomey-Calavi) et dans la Rivière Sô (Dogodo) pour voir s'ils sont pollués par les HAP pétrogéniques provenant des barques motorisées et du transport des produits pétroliers (figure 2).

Les échantillons d'eau pour l'analyse physico-chimique ont été prélevés à l'aide de flacons en plastique de 1,5 litre. Les prélèvements sont faits sur deux jours consécutifs : le premier jour entre $13 \mathrm{~h}$ et $19 \mathrm{~h}$ dans le lac Nokoué, puis le deuxième jour à la même heure dans la rivière Sô. Les flacons destinés au prélèvement en profondeur sont lestés et attachés à une ficelle de longueur suffisante pour atteindre la profondeur requise. Une fois remplis au $2 / 3$, ils sont hermétiquement fermés dans l'eau pour empêcher l'oxygène d'y pénétrer. Ils sont ensuite nettoyés et étiquetés. Les étiquettes portent les mentions suivantes : date, lieu et heure du prélèvement. Ils sont ensuite acheminés au laboratoire d'IRGIP AFRICA pour les analyses.

La récolte des poissons a été faite dans les captures issues de la pêche artisanale, à partir des filets. Les pêcheurs ont été sollicités pour la capture. Les échantillons de poissons pour l'analyse toxicologique ont été réalisés dans des bocaux en plastique. Les prélèvements sont faits sur deux jours consécutifs : le premier jour entre $13 \mathrm{~h}$ et $19 \mathrm{~h}$ sur le lac Nokoué, puis le deuxième jour à la même heure dans la rivière Sô. Les bocaux sont ensuite étiquetés. Les étiquettes portent les mentions suivantes : date, lieu et heure de prélèvement. Ils sont ensuite acheminés au laboratoire pour les analyses.

\subsection{Analyse et traitement des données}

Les analyses des échantillons d'eau et de poissons sont réalisées au laboratoire d'IRGIP-AFRICA. Le matériel utilisé pour la mesure des paramètres est constitué d'un $\mathrm{pH}$-mètre de marque HANNA pour la mesure du $\mathrm{pH}$ et de la conductivité ; la conductivité électrique a été mesurée avec un conductimètre de marque HANNA ; un colorimètre de marque DR 890 pour la mesure des nitrites et des sulfates.

Le dosage des HAP dans les poissons a été fait avec un chromatographe de marque Thermoscientific. Les échantillons ont permis d'étudier respectivement les paramètres physico-chimiques et toxicologiques d'eaux et de poissons. Pour les échantillons d'eau ; quatre (04) paramètres physico-chimiques ont été étudiés : le potentiel d'hydrogène, la conductivité, les nitrites et les sulfates. Pour les échantillons d'eau et de poissons, cinq 
paramètres toxicologiques ont été étudiés: le naphtalène, l'acénaphtène, l'anthracène, le fluoranthène et l'acénaphtylène

\subsubsection{Détermination des nitrites}

Plusieurs méthodes peuvent être utilisées pour le dosage des nitrites, à savoir : la méthode à la sulfanilamide, la méthode par flux contenu et la méthode au réactif de Zambelli. Utilisée dans le cadre de la présente étude, la méthode au réactif de Zambelli est basée sur la capacité de l'acide sulfurique à former en présence des ions nitrites en milieu chlorhydrique en présence d'ammoniaque pure et du phénol un complexe coloré en jaune dont l'intensité est proportionnelle à la concentration en nitrites et susceptible d'être mesuré par colorimétrie. Il faut prélever $50 \mathrm{ml}$ d'eau à analyser, ajouter $2 \mathrm{ml}$ du réactif de Zambelli. Ensuite il faut agiter et laisser au repos $10 \mathrm{mn}$ puis ajouter par la suite $2 \mathrm{ml}$ d'ammoniaque pure. Enfin il faut effectuer la lecture au spectrophotomètre à la longueur d'onde de 435 nm (Rodier , 2005).

\subsubsection{Détermination des sulfates}

Après avoir pipeté $25 \mathrm{ml}$ d'échantillon dans une cuvette propre (l'échantillon préparé), il faut remplir une autre cuvette avec $25 \mathrm{ml}$ d'eau désionisée (le blanc). Ensuite ajouter $1 \mathrm{ml}$ de réactif Sulfure 1 à chaque cuvette, agiter par la suite pour mélanger (utiliser le compte-gouttes gradué de $1 \mathrm{ml}$ ). Après cela, il faut ajouter $1 \mathrm{ml}$ de réactif Sulfure 2 à chaque cuvette, agiter immédiatement pour mélanger (une coloration rose se développe en présence de sulfure, la solution vire au bleu), et enfin placer le blanc dans le puits de mesure et lire la mesure.

\subsubsection{Dosage des HAP dans l'organisme des poissons}

Les échantillons de poissons ont été broyés avant le dosage. La méthode utilisée pour le dosage des HAP dans les poissons est la chromatographie en phase gazeuse couplée à un détecteur à ionisation de flamme (CG/FID). Quant aux échantillons d'eau, ils ont été directement dosés. Cette méthode de dosage se déroule en trois étapes. Extraction des HAP dans les poissons, le maintien des conditions chromatographiques et les facteurs de bioaccumulation.

\section{Extraction des HAP dans les poissons}

Après avoir broyé l'échantillon et prélevé précisément 5,00 g (balance avec une précision de $\pm 0,01 \mathrm{~g}$ ) dans un bécher de $50 \mathrm{ml}$, on y ajoute $5 \mathrm{ml}$ d'acétone à l'aide d'une pipette graduée. Le mélange est agité à l'aide d'un agitateur « vortex » jusqu'à l'obtention d'une bonne dispersion de l'échantillon. Ensuite, à l'aide d'une pipette graduée, une quantité exacte de 5 $\mathrm{ml}$ d'hexane est ajoutée et agitée avec l'agitateur « vortex ». Il faut ajouter 25 
ml d'eau et agiter à l'aide de l'agitateur « vortex » puis centrifuger à environ 1600 tour/min pendant 2 minutes. Prendre une aliquote de $2 \mathrm{ml}$ de la phase supérieure d'hexane et la transférer dans un tube de $15 \mathrm{ml}$ avec bouchon de téflon contenant $0,15 \mathrm{~g}$ de gel de silice. Par la suite, il faut agiter les tubes au moins 10 minutes au moyen du vortex et laisser déposer le gel de silice. Après ces étapes, il faut ensuite prélever le surnageant avec une pipette Pasteur et le transférer dans un micro flacon (vial) et enfin effectuer le dosage.

\section{Conditions chromatographiques}

Pour effectuer le dosage, le chromatographe doit être dans les conditions suivantes :

L'injecteur utilisé est sans chauffage (On column). Les caractéristiques de la

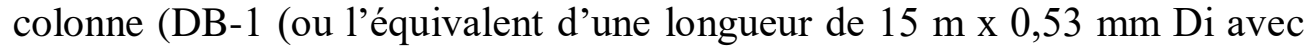
une phase stationnaire de 0,15 $\mathrm{m}$ Gaz vecteur : Hélium Débit visé : 5,0 $\mathrm{ml} / \mathrm{min}$ (visé $37 \mathrm{~cm} / \mathrm{s}$ )). Le four est programmé à une température initiale de $40^{\circ} \mathrm{C}$ durant 0,25 minute. Le taux du premier palier de programmation est de $30^{\circ} \mathrm{C}$ et le final à $300^{\circ} \mathrm{C}$. Concernant le deuxième palier, son taux est fixé à $10^{\circ} /$ minute avec une finale de $300^{\circ} \mathrm{C} /$ minute durant sept minutes. Le détecteur FID est maintenu à une température de $360^{\circ} \mathrm{C}$. Le volume d'injection est $1 \mu \mathrm{l}$.

\section{Facteur de bioaccumulation}

Lorsque les HAP pétrogéniques sont entrés dans l'organisme des Tilapia, ils ont tendance à s'accumuler. Au fil des temps, les concentrations de ces HAP pétrogéniques présents dans l’organisme deviennent plus élevées que dans son environnement immédiat. Cette bioaccumulation peut se quantifier par le facteur de bioaccumulation noté $F_{b}$. Il est calculé de la manière suivante :

$$
F b=\frac{\text { Taux de HAP dans le tilapia }}{\text { Taux de HAP dans l'échantillon d'eau }} \times 100
$$

Les données obtenues ont été traitées à l'aide des logiciels statistiques et d'analyses multi variées : le tableur EXCEL pour l'interprétation des tableaux à travers le tracé de graphique ; le logiciel MINITABß14 pour réaliser la matrice de corrélation entre les différentes variables utilisées. Les normes des paramètres physico-chimiques utilisées sont celles du code de la Santé Publique (2001). Pour les échantillons d'eau, la norme NF en ISO 17993 définie par le CIRC (2012) pour les HAP pétrogéniques a été utilisée. En ce qui concerne les Tilapia, il n'existe pas encore des normes définies pour les HAP pétrogéniques dans les poissons frais. 


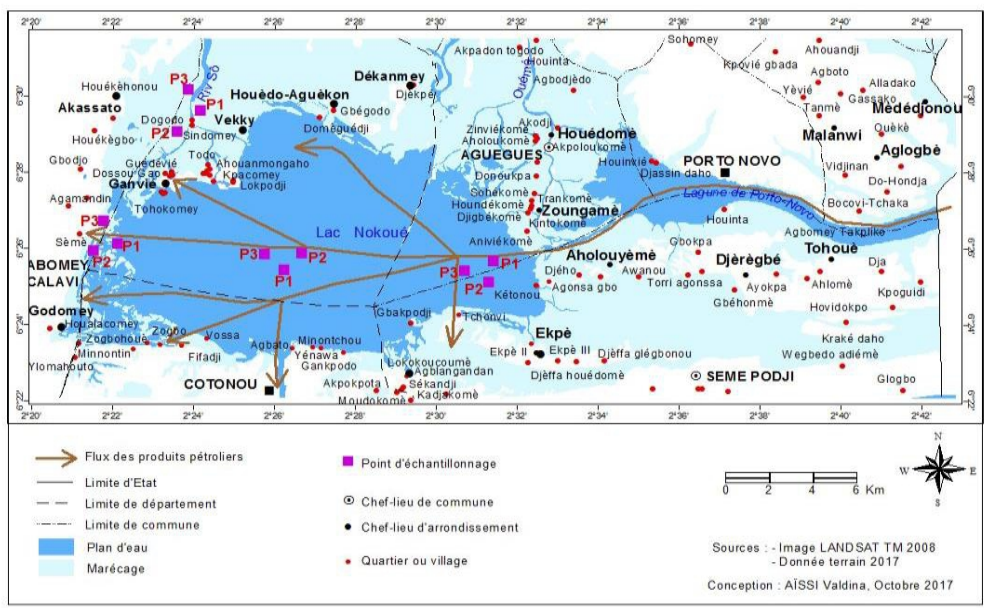

Figure 2: Trafic des produits pétroliers et de localisation des points de prélèvement

\section{Résultats}

\subsection{Caractères généraux des différentes espèces de Tilapia prélevés}

Les Tilapias sont les poissons les plus consommés par les populations béninoises d'où leur choix pour notre étude. Ils font partie de la famille des Cichlidae (Figure 3). Les espèces de cette famille se reconnaissent aisément par une tête portant une seule narine de chaque côté, un os operculaire non épineux, un corps comprimé latéralement, couvert essentiellement d'écailles, une longue nageoire dorsale à partie antérieure épineuse, et enfin une nageoire anale. Selon Zaatout (2007), les Tilapia possèdent plusieurs caractères biologiques favorables à l'élevage. Le régime alimentaire herbivore et omnivore permet une transformation efficace de l'énergie dans la chaîne trophique des écosystèmes aquatiques.

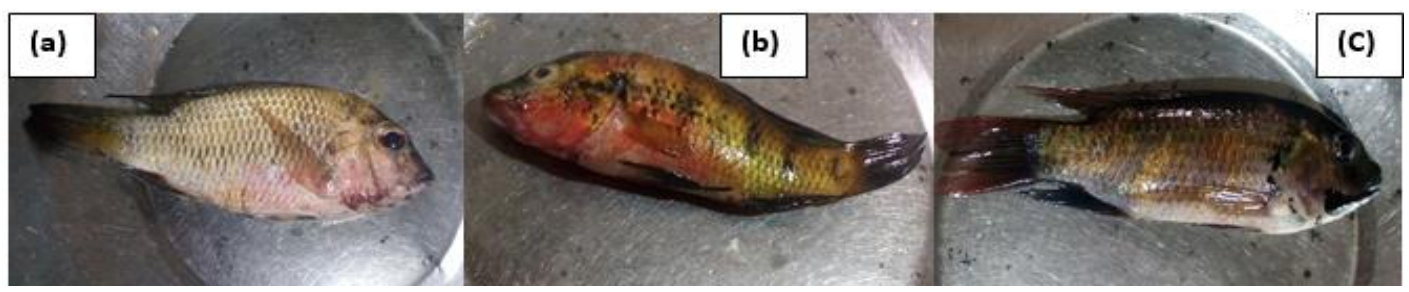

Figure 3: Différentes espèces de Tilapia prélevés dans le lac Nokoué. (a) Tilapia guineensis,

(b) Hemichromis fasciatus Hemichromis fasciatus, (c) Sarotherodon melanotheron.

Ces espèces tolèrent une large gamme de salinité et supportent des eaux de faible concentration d'oxygène dissous et sont peu sensibles aux maladies, au stress de capture et de manipulation.

S'adaptant aisément à la captivité, ils se reproduisent en élevage. Ils ont une croissance rapide. Selon leur morphologie, les Tilapia étaient classés 
en trois groupes : Tilapia (a), Sarotherodon (b), et Neotilapia (c). Cependant, Zaatout (2007) pense que de nos jours, le nombre d'espèces de Tilapia a fortement augmenté avec la découverte d'espèces nouvelles, ce qui a conduit à faire un dénombrement de plus de 90 espèces.

\subsection{Caractéristiques physico-chimique de l'eau du lac Nokoué}

Les résultats des paramètres physico-chimiques des échantillons d'eau sont présentés sur les graphiques suivants (Figure 4) :

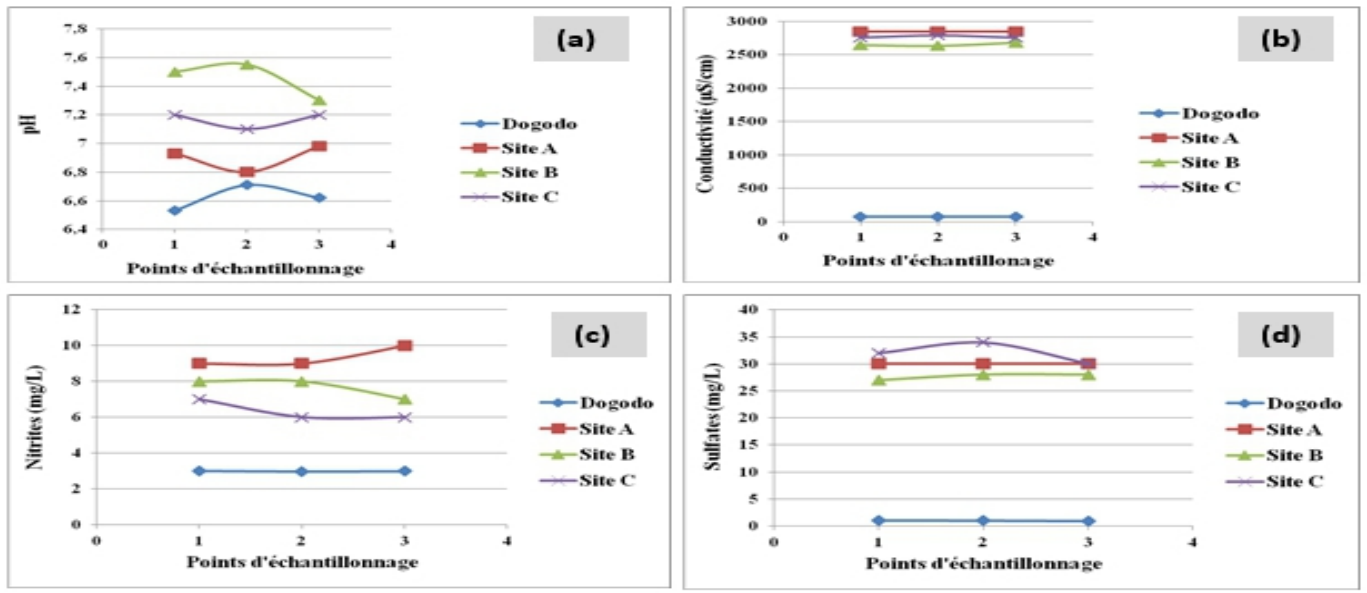

Figure 4 : Caractéristiques physico-chimiques des eaux du lac sur les sites de prélèvement. (a)Variation du $\mathrm{pH}$, (b) Variation de la conductivité, (c) Variation de la teneur en nitrites, (d) Variation de la teneur en sulfates.

Au niveau de la rivière Sô à Dogodo, le pH moyen est de 6,62 (Fig.4a) alors que sur le lac Nokoué, le pH moyen est de 6,90 à Kétonou (Site A) ; 7,45 sur le site $\mathrm{B}$ à $6 \mathrm{~km}$ de Domèguédji ; et de 7,17 à l'Embarcadère d'AbomeyCalavi. Le pH est donc plus élevé sur le lac Nokoué que dans la rivière Sô. Dans le lac, s'observe la même évolution de la conductivité moyenne que le $\mathrm{pH}$. Au niveau de la rivière Sô, à Dogodo, la conductivité moyenne est de $79,33 \mu \mathrm{S} / \mathrm{cm}$ (Fig.4b). Par contre, au niveau du lac Nokoué, elle est de 2841 $\mu \mathrm{S} / \mathrm{cm}$ à Kétonou (Site A) ; 2650,67 $\mu \mathrm{S} / \mathrm{cm}$ sur le site B à $6 \mathrm{~km}$ de Domèguédji et de $2766,33 \mu \mathrm{S} / \mathrm{cm}$ à 1 'Embarcadère (Site $\mathrm{C}$ ). La concentration des nitrites est plus élevée sur le lac Nokoué que dans la rivière Sô. Au niveau de la rivière Sô à Dogodo, la concentration moyenne des nitrites est de 2,99 mg/L (Fig.4c). Par contre, sur le lac Nokoué, la concentration moyenne des nitrites est de $9,33 \mathrm{mg} / \mathrm{L}$ à Kétonou (Site $\mathrm{A}) ; 7,67 \mathrm{mg} / \mathrm{L}$ sur le site $\mathrm{B}$ à $6 \mathrm{~km}$ de Domèguédji et de $6,33 \mathrm{mg} / \mathrm{L}$ à l'embarcadère (Site $\mathrm{C}$ ). Le même constat se fait au niveau de la concentration des sulfates. Au niveau de la rivière Sô, la concentration moyenne des sulfates est de $0,99 \mathrm{mg} / \mathrm{L}$ (Fig.4d) alors que sur le lac Nokoué, elle est de $30 \mathrm{mg} / \mathrm{L}$ à Kétonou (Site A) ; 27,67 mg/L sur le site $\mathrm{B}$ à $6 \mathrm{~km}$ de Domèguédji et de $32 \mathrm{mg} / \mathrm{L}$ à l'embarcadère d'Abomey-Calavi (Site C). La 
concentration des sulfates est donc plus élevée sur le lac Nokoué que dans la rivière Sô.

De ces différents résultats, on remarque que le $\mathrm{pH}$, la conductivité, les concentrations des nitrites et les concentrations des sulfates sont plus élevés dans le lac Nokoué que dans la rivière Sô.

\subsection{Résultat de l'analyse des HAP dans l'eau et dans l'organisme des poissons}

Les résultats des paramètres toxicologiques dans les échantillons d'eau sont présentés sur la figure 5.

A partir de la figure 5a, on note une absence totale de naphtalène, d'acénaphtène et de fluoranthène dans les échantillons prélevés à Dogodo sur la rivière Sô. On y retrouve $0,01 \mu \mathrm{g} / \mathrm{L}$ d'anthracène et $0,03 \mu \mathrm{g} / \mathrm{L}$ d'acénaphtylène. Il en ressort également que les taux moyens des HAP pétrogéniques dans les échantillons d'eau prélevés sur les sites sont de 0,06 $\mu \mathrm{g} / \mathrm{L}$ à Kétonou ; $0,062 \mu \mathrm{g} / \mathrm{L}$ sur le site $\mathrm{B}$ à $6 \mathrm{~km}$ de Domèguédji et de 0,06 $\mu \mathrm{g} / \mathrm{L}$ à l'embarcadère d'Abomey-Calavi. Les taux des HAP pétrogéniques sur le lac Nokoué sont supérieurs à ceux obtenus dans la rivière Sô. On peut donc dire que le lac Nokoué présente une toxicité élevée en HAP pétrogéniques.

Les résultats présentés dans la figure $5 \mathrm{~b}$ montrent une absence totale de naphtalène, d'acénaphtène et de fluoranthène $(0 \%)$ dans la chair des individus de Tilapia guineensis prélevés dans la rivière Sô à Dogodo. On retrouve des concentrations en anthracène $(0,001 \mu \mathrm{g} / \mathrm{L})$ et en acénaphtylène $(0,002 \mu \mathrm{g} / \mathrm{L})$. Les taux moyens de HAP pétrogéniques retrouvés dans la chair de Tilapia guineensis collectés dans le lac Nokoué sont de $0,0056 \mu \mathrm{g} / \mathrm{L}$ à Kétonou (Site A) ; de $0,0068 \mu \mathrm{g} / \mathrm{L}$ sur la Site B à $6 \mathrm{~km}$ de Domèguédji et de $0,0061 \mu \mathrm{g} / \mathrm{L}$ à l'embarcadère d'Abomey-Calavi. Il y a donc un faible taux de toxicité en HAP pétrogéniques chez Tilapia guineensis dans la rivière Sô et un taux élevé de toxicité en HAP pétrogéniques chez Tilapia guineensis dans le lac Nokoué. 

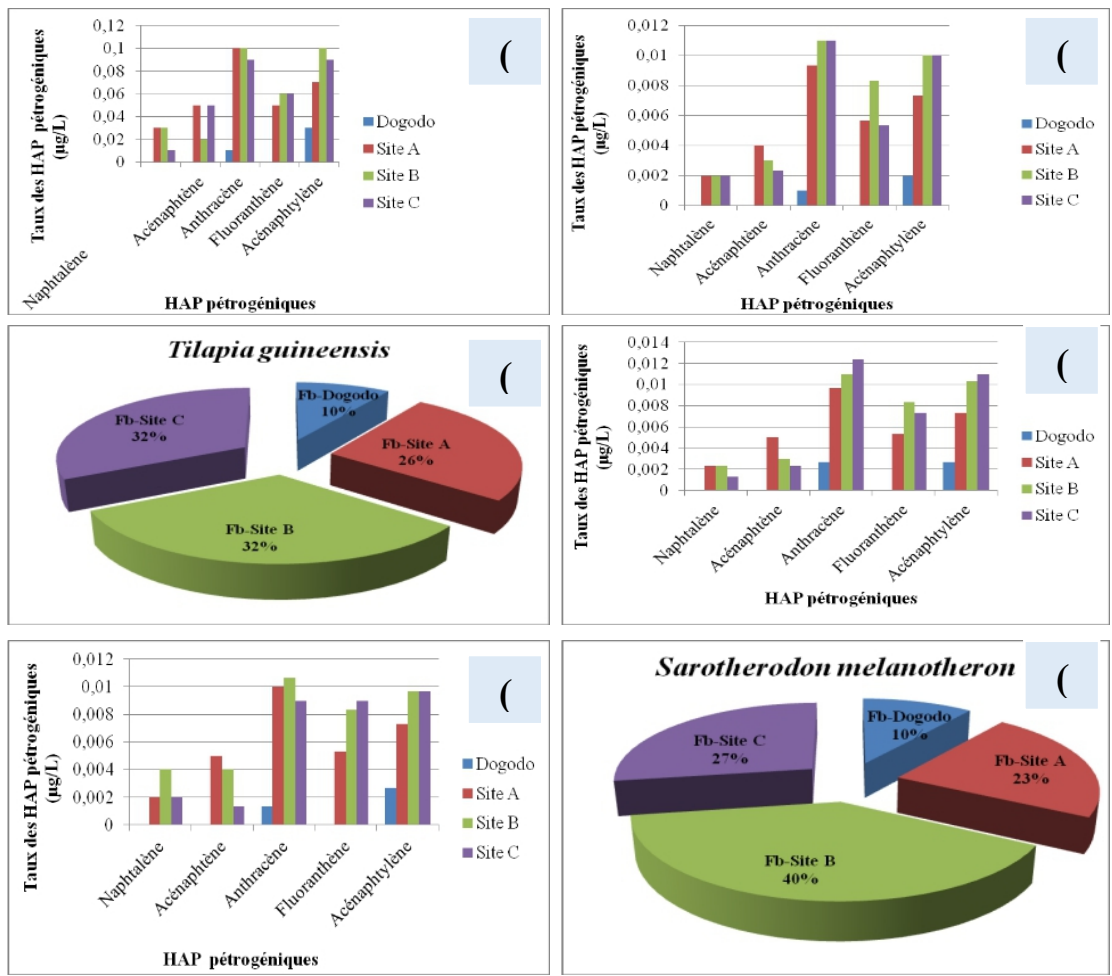

Figure 5 : Caractéristiques toxicologiques de l'eau du lac. (a) variation des taux des HAP pétrogéniques des échantillons d'eau, (b) variation des taux des HAP pétrogéniques obtenus chez Tilapia guineensis, (c) facteurs de bioaccumulation chez Tilapia guineensis, (d) variation des taux des HAP pétrogéniques obtenus chez Hemichromis fasciatus, (e) taux des HAP $(\mu \mathrm{g} / \mathrm{L})$ obtenus chez Sarotherodon melanotheron, (f) facteurs de bioaccumulation chez Sarotherodon melanotheron.

De la figure $5 \mathrm{~d}$, on constate une absence totale de naphtalène, d'acénaphtène et de fluoranthène chez les individus de Hemichromis fasciatus collectés dans la rivière Sô à Dogodo, mais on remarque la présence d'anthracène et de fluoranthène dans les mêmes proportions $(0,0026 \mu \mathrm{g} / \mathrm{L})$. On note également une toxicité des 5 HAP pétrogéniques chez les individus de Hemichromis fasciatus prélevés dans le lac Nokoué avec des valeurs moyennes de $0,0059 \mu \mathrm{g} / \mathrm{L}$ à Kétonou, de $0,007 \mu \mathrm{g} / \mathrm{L}$ sur le Site B à $6 \mathrm{~km}$ de Domèguédji, et de $0,0068 \mu \mathrm{g} / \mathrm{L}$ à l'embarcadère. Il y a un faible taux de toxicité en HAP pétrogéniques chez les individus de Hemichromis fasciatus collectés dans la rivière Sô et un taux élevé de toxicité en

HAP pétrogéniques chez les individus de Hemichromis fasciatus collectés dans le lac Nokoué.

De la figure $5 \mathrm{e}$, on constate une absence totale de naphtalène, d'acénaphtène et de fluoranthène chez les individus de Sarotherodon melanotheron collectés dans la rivière Sô à Dogodo, mais on remarque la présence de $0,0013 \mu \mathrm{g} / \mathrm{L}$ d'anthracène et de $0,0026 \mu \mathrm{g} / \mathrm{L}$ de fluoranthène. La 
figure montre également une toxicité des 5 HAP pétrogéniques chez les individus de Sarotherodon melanotheron prélevés dans le lac Nokoué avec des valeurs moyennes de $0,0059 \mu \mathrm{g} / \mathrm{L}$ à Kétonou, de $0,0073 \mu \mathrm{g} / \mathrm{L}$ sur le Site B à $6 \mathrm{~km}$ de Domèguédji, et de $0,0062 \mu \mathrm{g} / \mathrm{L}$ à l'embarcadère. Il y a un faible taux de toxicité en HAP pétrogéniques chez les individus de Sarotherodon melanotheron collectés dans la rivière Sô et un taux élevé de toxicité en HAP pétrogéniques chez les individus de Sarotherodon melanotheron collectés dans le lac Nokoué.

Les résultats obtenus lors du calcul du facteur de bioaccumulation sont présentés dans la figure 5f. Elle montre que les individus de Sarotherodon melanotheron prélevés dans la rivière Sô à Dogodo sont contaminés en HAP pétrogéniques à $10 \%$; à $23 \%$ à Kétonou, à $40 \%$ sur le site $\mathrm{B}$ à $6 \mathrm{~km}$ de Domèguédji et à $27 \%$ à l'embarcadère. Les individus de Sarotherodon melanotheron collectés dans le lac Nokoué ont accumulé plus de HAP pétrogéniques que ceux collectés dans la rivière Sô. Les individus de Sarotherodon melanotheron collectés dans le lac Nokoué sont plus contaminés en HAP pétrogéniques

\subsection{Corrélation entre les variables physico-chimiques et toxicologiques}

Le tableau II montre les coefficients de corrélation entre les différents paramètres mesurés. Les résultats montrent que le $\mathrm{pH}$ et la conductivité ne sont pas corrélés, ils ont une corrélation de 0,525 et une probabilité de 0,060. Il en est de même pour les nitrites et le $\mathrm{pH}$ qui ont une corrélation de -0,702 et une probabilité de 0,062 ; ainsi que pour les nitrites et la conductivité qui ont une corrélation de $-1,000$ et une probabilité de 0,089 . Le naphtalène est fortement corrélé avec le $\mathrm{pH}$, la conductivité et les nitrites respectivement avec des probabilités de 0,$014 ; 0,009$ et 0,007 . Par contre le naphtalène et les sulfates ne sont pas corrélés car la probabilité $(0,076)$ dépasse le seuil de significativité $(5 \%)$. L'acénaphtène est fortement corrélé avec le $\mathrm{pH}$, la conductivité, les nitrites et les sulfates avec des probabilités allant de 0,000 à 0,044 . L'anthracène est fortement corrélé avec la conductivité, les nitrites et les sulfates avec les mêmes probabilités $(0,000)$ et n'est pas corrélé avec le pH avec une probabilité de 0,195 . Le fluoranthène est fortement corrélé avec le $\mathrm{pH}$, la conductivité et les nitrites avec une probabilité moyenne de 0,011 et n'est pas corrélé avec les sulfates $(0,187)$. L'acénaphtylène est corrélé avec le $\mathrm{pH}$, la conductivité, les nitrites et les sulfates avec une probabilité moyenne de 0,012. Les 5 HAP pétrogéniques sont tous corrélés entre eux avec une corrélation moyenne de 0,78 et une probabilité moyenne de 0,011. 
Tableau II : Matrice de corrélation entre les variables Physico-Chimiques et les HAP

\begin{tabular}{|c|c|c|c|c|c|c|c|c|c|c|}
\hline Paramètres & & $\mathrm{pH}$ & Cond & NO2- & SO4- & Naphtalène & Acénaphtène & Anthracène & Fluoranthène & $\begin{array}{l}\text { Acénaphty } \\
\text { lène }\end{array}$ \\
\hline \multicolumn{11}{|l|}{$\mathbf{P h}$} \\
\hline \multirow[t]{2}{*}{ Conductivité } & $\mathbf{r}$ & 0,525 & 1 & & & & & & & \\
\hline & $\mathbf{p}$ & 0,060 & & & & & & & & \\
\hline \multirow[t]{2}{*}{$\mathrm{NO}_{2}^{-}$} & $\mathbf{r}$ & $-0,702$ & $-1,000$ & 1 & & & & & & \\
\hline & $\mathbf{p}$ & 0,062 & 0,089 & & & & & & & \\
\hline \multirow[t]{2}{*}{$\mathrm{SO}_{4}^{-}$} & $\mathbf{r}$ & 0,584 & 0,997 & 0,987 & 1 & & & & & \\
\hline & $\mathbf{p}$ & 0,043 & 0,000 & 0,000 & & & & & & \\
\hline \multirow[t]{2}{*}{ Naphtalène } & $\mathbf{r}$ & 0,605 & 0,845 & 0,875 & - & 1 & & & & \\
\hline & $\mathbf{p}$ & 0,014 & 0,009 & 0,007 & 0,913 & & & & & \\
\hline \multirow[t]{2}{*}{ Acénaphtène } & $\mathbf{r}$ & 0,566 & 0,989 & 0,848 & 0,998 & 0,998 & 1 & & & \\
\hline & $\mathbf{p}$ & 0,044 & 0,000 & 0,003 & 0,000 & 0,000 & & & & \\
\hline \multirow[t]{2}{*}{ Anthracène } & $\mathbf{r}$ & 0,422 & 0,976 & 0,946 & 0,969 & 0,978 & 0,834 & 1 & & \\
\hline & $\mathbf{p}$ & 0,195 & 0,000 & 0,000 & 0,000 & 0,000 & 0,004 & & & \\
\hline \multirow[t]{2}{*}{ Fluoranthène } & $\mathbf{r}$ & 0,724 & 0,861 & 0,959 & 0,456 & $\mathbf{0 , 8 1 9}$ & 0,746 & 0,986 & 1 & \\
\hline & $\mathbf{p}$ & 0,024 & 0,008 & 0,000 & 0,187 & 0,007 & 0,034 & 0,000 & & \\
\hline \multirow[t]{2}{*}{ Acénaphtylène } & $\mathbf{r}$ & 0,698 & 0,984 & 0,954 & $\mathbf{0 , 8 1 2}$ & 0,767 & 0,611 & 0,957 & 0,997 & 1 \\
\hline & $\mathbf{p}$ & 0,043 & 0,000 & 0,000 & 0,006 & 0,028 & 0,042 & $\mathbf{0 , 0 0 0}$ & $\mathbf{0 , 0 0 0}$ & \\
\hline
\end{tabular}

$\mathrm{r}$ : valeur de corrélation ; $\mathrm{p}$ : valeur de probabilité ; seuil de significativité $: \mathrm{r}$ supérieur ou égale à 0,5 et $p$ inférieur

\section{Discussion}

Les valeurs de $\mathrm{pH}$ obtenus dans les échantillons d'eau prélevés sur la rivière Sô $(6,53-6,71)$ et sur le lac Nokoué $(6,8-7,55)$ respectent les normes de qualité admises fixées par le CIRC (2012) dont les valeurs sont comprises entre 6,5 et 8,5. Les études récentes (Gbesso et al, 2015) ont rapporté des valeurs de $\mathrm{pH}$ presque similaires $(7,58-7,95)$. Ces valeurs de $\mathrm{pH}$ seraient favorables au développement des espèces aquatiques.

Les valeurs moyennes de la conductivité obtenues dans les échantillons d'eau prélevés à Dogodo dans la rivière Sô $(79-80 \mu \mathrm{s} / \mathrm{Cm})$ ne dépassent pas la norme de qualité des eaux admises par le CIRC (2012) dont les valeurs sont comprises entre 180 et $1000 \mu \mathrm{s} / \mathrm{Cm}$. Les valeurs de la conductivité électrique obtenues dans les échantillons d'eau prélevés sur les Sites A, B et C du lac Nokoué $(2630$ - $2841 \mu \mathrm{s} / \mathrm{Cm})$ sont supérieures à la norme de qualité admise (180-1000 $\mu \mathrm{s} / \mathrm{Cm})$. Par ailleurs en 2008, Dovonou a rapporté des valeurs de conductivité comprises entre 1025 et $1073,3 \mu \mathrm{s} / \mathrm{Cm}$. Les valeurs obtenues indiquent que le milieu est pollué. L'eau du lac Nokoué serait inapte aux espèces aquatiques (en particuliers les Tilapia) et à la plupart des usagers et pourrait constituer une menace pour la santé publique et l'environnement. 
Les concentrations de nitrites obtenues à Dogodo dans la rivière Sô (2,98-3 mg/L) et sur les Sites A, B et C du lac Nokoué (6-10 mg/L) sont supérieures à la limite admise du code de la santé Publique (2001) qui est de $0,5 \mathrm{mg} / \mathrm{L}$. En 2014, Dovonon et al. ont rapportés des valeurs de nitrites comprises entre 0,04 et $0,6 \mathrm{mg} / \mathrm{L}$. Ces valeurs sont pour la plupart supérieures à la norme internationale $(0,5 \mathrm{mg} / \mathrm{L})$. Ceci indique un état de pollution critique. En effet, une eau renfermant des nitrites même à faibles doses peut être considérée comme suspecte voire létale pour les poissons. Ceci peut provoquer des problèmes d'eutrophisation pour le lac et entraîner une diminution de la capacité de reproduction des espèces aquatiques selon Gaujous (1995).

Les concentrations en sulfates obtenues comprises entre 0,97 et $1 \mathrm{mg} / \mathrm{L}$ à Dogodo sur la rivière Sô et comprises entre 27 et $34 \mathrm{mg} / \mathrm{L}$ sur les Sites A, $\mathrm{B}$ et $\mathrm{C}$ du lac Nokoué sont semblables à celles obtenues par Waris (2010) et ne dépassent pas la limite admise par la législation du code de la santé Publique qui est de $250 \mathrm{mg} / \mathrm{L}$. Les sulfates ont tendance à se déposer au fond de l'eau, c'est-à-dire dans les sédiments. C'est ce qui expliquerait la faible concentration des sulfates obtenue de façon générale au niveau de tous les points de prélèvement.

Les résultats des taux des HAP pétrogéniques obtenus au niveau des échantillons d'eau prélevés ont permis de constater une absence total de naphtalène, d'acénaphtène et de fluoranthène à Dogodo dans la rivière Sô. Toutefois la concentration d'anthracène trouvée $(0,01 \mu \mathrm{g} / \mathrm{L})$ respecte la norme admise des HAP pétrogéniques $(0,01 \mu \mathrm{g} / \mathrm{L})$; quant à la concentration d'acénaphtylène présente $(0,03 \mu \mathrm{g} / \mathrm{L})$, elle dépasse la norme $\mathrm{NF}$ en iso définie par le CICR (2012) qui est de 0,001 $\mu \mathrm{g} / \mathrm{L}$. Sur les Sites A, B et C du lac Nokoué, les valeurs des concentrations des 5 HAP pétrogéniques obtenus $(0,01$ à $0,03 \mu \mathrm{g} / \mathrm{L}$ pour le naphtalène ; 0,02 à $0,05 \mu \mathrm{g} / \mathrm{L}$ pour l'acénaphtène ; 0,09 à $0,1 \mu \mathrm{g} / \mathrm{L}$ pour l'anthracène ; 0,05 à $0,06 \mu \mathrm{g} / \mathrm{L}$ pour le fluoranthène et 0,07 à $0,1 \mu \mathrm{g} / \mathrm{L}$ pour l'acénaphtylène) dépassent la norme admise $(0,001 \mu \mathrm{g} / \mathrm{L}$ pour le naphtalène, l'acénaphtène, le fluoranthène, l'acénaphtylène et 0,01 $\mu \mathrm{g} / \mathrm{L}$ pour l'anthracène). Les concentrations des HAP pétrogéniques sur le lac Nokoué sont supérieures à celles obtenus sur la rivière Sô. Tossou (2001) a rapportée des concentrations de HAP comprises entre 0,067 et 0,137 $\mu \mathrm{g} / \mathrm{L}$, confirmant ainsi les résultats de notre étude.

On peut donc conclure que le lac Nokoué présente une toxicité élevée en HAP pétrogéniques. Ceci témoigne des conséquences directes de la forte pression anthropique exercée sur le lac. L'accumulation des HAP pétrogéniques et leur dégradation et/ou transformation dans le lac ont des influences à court ou à long terme sur les poissons et les populations qui exploitent et utilisent cette ressource naturelle. 
Les trois espèces de Tilapia collectés dans le lac Nokoué ont accumulé plus de HAPs pétrogéniques que les Tilapia collectés dans la rivière Sô. Ce sont donc des bioaccumulateurs de pollution. La qualité des Tilapia retrouvés dans la rivière Sô est meilleure à celle des Tilapia du lac Nokoué. Les HAP pétrogéniques retrouvés dans l'organisme des Tilapia sont susceptibles de les étouffer, diminuer leur croissance, réduire la longueur de leur taille et diminuer leur poids. Ces HAP pétrogéniques peuvent également avoir des conséquences sur leur comportement, leur alimentation et leur reproduction Baussan (2000).

La matrice de corrélation a permis d'observer de fortes corrélations entre les différentes variables (corrélation supérieure à 0,5 et probabilité inférieure à 5\%). Ainsi, certains paramètres toxicologiques peuvent influencer les paramètres physico-chimiques. Les fortes corrélations obtenues entre les différentes variables physico-chimiques et toxicologiques signifient que lorsque les concentrations en naphtalène et en fluoranthène augmentent, le $\mathrm{pH}$, la conductivité et les nitrites aussi augmentent. Lorsque la concentration en anthracène augmente, la conductivité, les nitrites et les sulfates aussi augmentent. Lorsque l'acénaphtène et l'acénaphtylène augmentent le $\mathrm{pH}$, la conductivité, les nitrites et les sulfates également augmentent. Les faibles corrélations obtenues montrent que les sulfates diminuent quand le naphtalène et le fluoranthène augmentent (respectivement $-0,913$ et 0,456 comme corrélation) et vice-versa. Le $\mathrm{pH}$ diminue lorsque la concentration en anthracène augmente $(0,422)$. Tossou (2001) a rapportée des valeurs de corrélation et de probabilité entre les paramètres physico-chimiques et les HAP comprises entre 0,808 et 0,999 . La présence des HAP pétrogéniques dans le milieu lacustre influence les paramètres physico-chimiques à savoir : le pH, la conductivité, les sulfates et les nitrites.

\section{Conclusion}

Les résultats des recherches effectuées dans la présente étude visant à évaluer les conséquences directes de la pression anthropique par le transport des hydrocarbures sur le lac Nokoué ont révélé que l'eau du lac Nokoué est de qualité physico-chimique douteuse pour la vie des espèces présentes. Les espèces de poissons Tilapia du lac Nokoué sont exposées à la pollution par les HAP pétrogéniques. Cette contamination est révélée par l'accumulation des polluants dans la chair des poissons échantillonnés et Il y a une forte corrélation entre les composantes physico-chimiques de l'eau et les HAP pétrogéniques présents dans l'eau. Ceci témoigne des conséquences directes de ces contaminants sur la qualité de l'eau du lac Nokoué. En vue de contribuer à la réduction de la pollution du lac Nokoué en HAP pétrogéniques, les suggestions suivantes sont faites à l'endroit des autorités politicoadministratives chargées de la gestion des ressources en eau au Bénin : 
- sensibiliser les pêcheurs et les conducteurs de barques motorisées sur l'intérêt d'utiliser des barques non motorisées et supprimer l'utilisation des barques motorisées qui polluent le lac avec les hydrocarbures.

- veiller à l'interdiction du transport et de la commercialisation des produits pétroliers sur le lac. Il faut en faire de même des rejets d'huile de vidange dans le lac Nokoué ;

- procéder à la dépollution du lac pour permettre la prolifération des espèces animales aquatiques telles que les poissons, les crevettes, les huîtres ;

- réaliser l'étude des HAP pétrogéniques sur d'autres espèces aquatiques autre que les poissons Tilapia afin d'évaluer également leur impact sur ces derniers et sur les sédiments aussi ;

- réaliser l'étude des 11 HAP restants afin d'évaluer leur impact sur les espèces aquatiques ;

- fixer des normes pour tous les HAP aussi bien dans les poissons fumés que dans les poissons frais car le lac Nokoué est la plus grande réserve de protéines animales du Sud-Bénin.

\section{References:}

1. Ahoudi H, Gnandi K, Tanouayi G, OuroSama K. (2015). "Caractérisation Physico-chimique et état de pollution par les élements traces métalliques des eaux souterraines de Lomé (Sud Togo) : cas du quartier Agoe Zongo". Larhyss Journal, 24: 41-56.

2. Anderson C., (2007), " Guide synthèse : élaboration d'un plan directeur de bassin versant de lac et adoption de bonnes pratiques ", 32 p.

3. Anfray C., Vaillant E., (2016), 'Ecologie et pollution: les deux visages de la Chine", 60p.

4. Baechler L., (2015), " La pollution de l'eau : problème clé de la gestion de la ressource, diplôme du cycle 2" 60p.

5. Baussant, T., (2000), " PAH metabolites in bile, cytochrome P4501A and DNA adducts as environmental risk parameters for chronic oil exposure", 241-258p.

6. Centre International de Recherche sur le Cancer, 2012, "Art: Définition des termes liés au cancer", $15 \mathrm{p}$

7. Dovonou F., (2008), " Pollution des plans d'eau du sud Benin et risques écotoxicologiques : cas du lac Nokoué ", DEA, 70 p.

8. Dovonou E. F. (2012). "Diagnostic qualitatif et environnemental de l'aquifère superficiel du champ de captage intensif de Godomey au Bénin (Afrique de l'Ouest) : éléments pour un plan d'actions stratégiques de protection des ressources en eau souterraine 
exploitées". Doctorat ès Science en Hydrologie et Gestion Intégrée des Ressources en Eau. Université d'Abomey, Bénin, p.143

9. Dovonon L., Saizonou M., Youssao A., Gbaguidi M., Soclo H., Sohounhoule D., (2014), " Contribution des eaux usées ménagères et des eaux de ruissellement dans la pollution du lac Nokoué au Bénin"

10. Fiogbe E., Boko M., Badahoui A., (2009), "Art : les causes de la dégradation du chenal de Cotonou", 13p.

11. Gaujous D., (1995), "La pollution des milieux aquatiques (Aidemémoire), Paris"', $74 \mathrm{p}$.

12. Gunther A., (1866), "Poissons d'eaux douces et saumâtres de basse Guinée", 805 p.

13. Gbesso F., Akognongbe A., et Vissin E., 2015, "Contamination de tilapia guineensis par le cadmium dans le chenal de cotonou", $20 \mathrm{p}$.

14. Hounsinou P, Mama D, Dovonou F. Alasane A. (2015). "Seasonal evolution of the quality microbiological of the natural waters in the township of Abomey-Calavi (South Benin) ". British Journal of Earth Sciences Research, 3(1): 30-41.

15. Hoteyi SMI, Gnimadi CC, Adjadji GV, Igoué AM, Mensah GA. (2014). "Analyse des risques de consommation des eaux en sachet pour les populations dans ville de Porto-Novo au Sud-Bénin. " Bulletin de la Recherche Agronomique du Bénin (BRAB). Numéro spécial Agrobiodiversité et Santé publique, p. 8.

16. Roussel, (2002), "Contribution à l'étude de la répartition des HAP entre les phases gazeuses et particulaires", 22p

17. Tossou E., (2001), "Impact du trafic des produits pétroliers sur les écosystèmes lacustres: cas du lac Nokoué et de la lagune de Cotonou"'111 p.

18. Waris C, Daouda M, François A, (2010)a. " Etude des variations spatio-temporelles de la pollution des eaux de la lagune de PortoNovo. Int. J. Biol. Chem. Sci., 4(4)1017-1029.

19. Welcomme R., (1971), "Evolution de la pêche intérieure, Son état actuel et ses potentialités. FAO. Rome", 97 p.

20. Zaatout R., (2007), "Effet du régime alimentaire sur la croissance des alevins de Tilapia", $77 \mathrm{p}$. 